\title{
A COLLECTION SCHEME FOR TRACING INFORMATION OF PIG SAFETY PRODUCTION
}

\author{
Qingyao Luo ${ }^{1,2}$, Benhai Xiong ${ }^{1,2, *}$, Liang Yang ${ }^{1,2}$ \\ ${ }^{1}$ Institute of Animal Sciences, Chinese Academy of Agricultural Sciences, Beijing 100193, \\ China; \\ ${ }^{2}$ State Key Laboratory of Animal Nutrition, Beijing 100193, China \\ * Corresponding author, Address: Institute of Animal Sciences, Beijing 100193, China, \\ Tel:+86-10-62816017, Fax:+86-10-62815988,Email: bhxiong@iascaas.net.cn
}

Abstract: $\quad$ This study takes one main production pattern of smallhold pig farming in Tianjin as a study prototype, deeply analyzes characters of informations about tracing inputs including vaccines, feeds,veterinary drugs and supervision test in pig farming, proposes inputs metadata, criteria for integrating inputs event and interface norms for data transmision, developes and completes identification of 2D ear tags and traceability information collection system of pig safety production based on mobile PDA. The system has implemented functions including setting and invalidate of 2D ear tags, collection of tracing inputs and supervision in the mobile PDA and finally integration of tracing events (the epidemic event,feed event,drug event and supervision event) on the traceability data center (server). The PDA information collection system has been applied for demonstration in Tianjin, the collection is simple, convenient and feasible. It could meet with requirements of traceability information system of pig safety production.

Key words: Pig; PDA; Traceability; Information Collection

\section{INTRODUCTION}

The establishment of animal identification and disease traceability system is an urgent demand for effectively preventing and controling major animal diseases, insuring animal-derived food security and promoting the sustained and healthy development of animal husbandry.Because mainbodies engaged

Please use the following format when citing this chapter:

Luo, Q., Xiong, B. and Yang, L., 2009, in IFIP International Federation for Information Processing, Volume 295, Computer and Computing Technologies in Agriculture II, Volume 3, eds. D. Li, Z. Chunjiang, (Boston: Springer), pp. 2239-2247. 
in rearing, slaughter, movement of pigs have poor organization, unsubstantial information infrastructure, collect and manage data mainly by hand, and these data collections are characterred by scatterred sites, long average cycles, low rate, small volume data and poor collection environments (Wang,2007), therefore, in order to improve quality and safety of the traceability data collection, to reduce cost and to improve the running efficiency, it is very essential to study optimizing scheme of information collection in pig safety traceability system. Since mad cow disease occurred in Britain in 1986, avian influenza and other zoonoses, Sudan, inferior milk powder and other food safety incidents sometimes occurred, food safety issues have aroused people's great concerns (Chen et al.,2007; Zhang,2006). The EU,Canada, the United States, Japan, Brazil, Australia and New Zealand and other countries have begun to implement animal identification tracking system(Zhang et al.,2008; Xiong et al.,2007; Ammendrup et al.,2001; Jill et al.,2005; N schembti et al.,2007; Caja et al.,2005).Studies for traceability system in China began in 2002 and have gradually constituted a number of relevant standards and guidelines such as "product traceability general specification", "traceabilty guide for beef products" constituted by Chinese goods code center, "the Administrative Rules on identification and rearing files for animal and poultry" (the Administrative Rules) issued by the Ministry of Agriculture on June 1, 2007(Decree No 67,2006). "Animal traceability system" led by the Ministry of Agriculture has been demonstrating in Beijing, Shanghai, Sichuan and Chongqing, in which main adopted technologies include 2D bar code identification and read technologies. The 2D bar code were used for animal identification, IC card as the interim carrier of animal quarantine and epidemic prevention data(Chen et al.,2007; Zhang,2006).

\section{MATIERIALS AND METHOD}

\subsection{Study Object}

This study takes smallhold farmers in Tianjin as a study prototype, takes commercial pigs and their products as study objects, takes rearing files contents stipulated by the Administrative Rules as basic reqiurements of information collection, carrys out the solution of information collection and management for pig safety traceability system. 


\subsection{Traceability Ear Tag for Pigs}

Complied with the animal traceability system led by the Ministry of Agriculture, this study adopts 2D ear tags for pigs which basic function is to track and identify pigs and their products. Ear tags are made of special plastic materials which conform to food contact safety regulations and do not result in product pollution and are set on the left or right ear. As show in Fig 1 , on the surface of ear tags there print identification numbers saw by the eyes and engrav 2D bar code image identified by the $2 \mathrm{D}$ bar code reader.

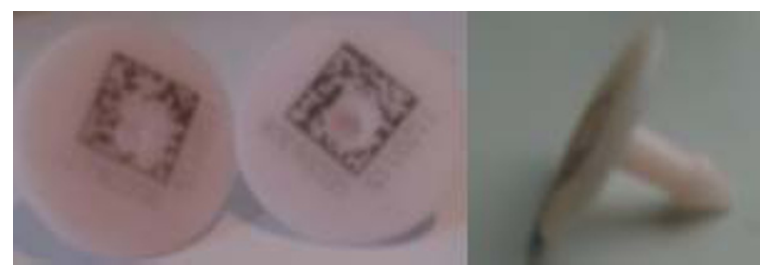

Fig. 1 the special ear tags with 2D bar code

Code rules of ear tags is the important basis of achieving general search and identification in all sections of the traceability system.According to identification code rules of animals stipulated by the Administrative Rules, identification code of the pig is consists of 15 digits, the first digit represents the type of livestock ("1" for pigs), the next six $\operatorname{digits}(2$ to 7 ) the administrative region code of the county (city) in accord with GB T22601999 (Nov. 31,2006) (Decree No 67,2006), the final 8 digits is the sequence which is the sequence order number in the same district. For example, the code like "1 12011500006306 " represents that the type is the pig, the farmimg place locates at Baodi District in Tianjin (administrative region code is 120115) and the individual order number is the six thounsands three hundreds and sixth in the scope of Baodi district.

\subsection{Identification Device for Ear Tags}

In order to strengthen the safety management of pig identification and improve the read efficiency of identification devices, the administrative office of animal traceability proposes the standard norm of traceability $2 \mathrm{D}$ ear tag used for pigs. Pig identification codes are converted to relative 2D code image according to the norm and engraved in the upper surface of ear tags through laser.On the surface of ear tags, there have both 15 digits code discerned by naked eyes and the corresponding 2D bar code image automatically identified by identification devices with speacial decode library for 2D bar code by scanning image.The Administrative office takes public bidding and has selected two kinds of smart mobile PDA with automatic identification for 2D pig ear tags (Fig 2). As show in Fig 2, the left 
smart PDA reader is based on the windows mobile 5.0 operation system and the right based on the Linux operation system.
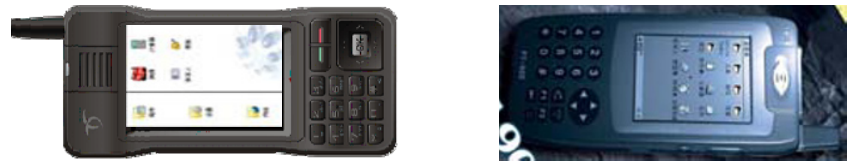

Fig.2 Intelligent PDA ear tag reader

\subsection{Collection and Management of Ttraceabilty Information}

\subsubsection{Identification of Ear Tags}

Based on PDA devices with the windows mobile 5.0, the study carries out to identify traceability ear tags by the P / Invoke quoting the library functions including API functions in coredll.dll, Mobile5DLL.dll, scanning functions in HWCamera.dll for PDA camera and the decoding library for 2D ear tags of pigs. In order to prevent and reduce unanticipated scanning, the system loads functions with scanning and identification just in modules required to identify pig ear tags. The solution can identify both standard 1D or 2D bar code through the camera and pig ear tag with especial 2D bar code and display the identified code number in the ear-tag information textbox. If the code number is repeated, the system gives a warning sound and does not show the code number in the textbox.

\subsubsection{Collection of Traceabilty Inputs}

Traceability Inputs in the process of pig farming including vaccines, feeds, veterinary drugs are the major information in pig product safety traceability system. Use informations of pig feeds and vaccines are registerred by group (pen) whereas veterinary drug registerred by head of pig. But in some circumstances, feeds and vaccines data can also record every pig and the veterinary drug data records by group.

In order to reduce the labor intensity for PDA operators, there are two ways to register informations of feeds and (or) veterinary drugs: Firstly, after registration of the epidemic prevention immediately to register uses of feeds and (or) veterinary drugs, the system automatically load the information of the required identity inforamtion of pig owner (owner, type of livestock, village, number of ear tags, ID of ear tags) which can not be amended. Secondly the registration of feeds and (or) veterinary drugs separating from 
registration of the epidemic prevention will need identify ear tags and fill out the required identity inforamtion of pigs owner.

In order to monitor and eradicate use of illicit drugs, veterinary administratives carry out occasional sampling supervision in process of rearing, transport and before-slaughtering of pigs. Test items mainly include clenbuterol hydrochloride, ractopamine, sulfa drugs.Testing methods are respectively test strip for rapid detection, ELISA and GC-MS. If testing result is positive, the sampling pig do not pass the supervision test.Whether positive or not, results record by head of pigs and transmit to the traceability data center. If the sampling result is unqualified, the group pigs including the sampling pig are regarded as ineligible. For inforamtion queries, query results show the last testing result by group. Supervision testing can detect a number of supervision items at one time.

\subsubsection{Transmission of Traceabilty Information}

All informations will be processed and finally transmitted to the data center.Traceabilty data can be saved to the local SQL Server CE database or text files or XML files in the PDA and text files in smart IC cards connected with the PDA. Once the network works normally, the system timely uploads informations to the traceabilty data center by GPRS. This study proposes metadata standards for traceabilty inputs, integrating norms for traceabilty events, interface specifications for data transmission and realizes the transmission solution of data updating and downloading between PDA and the data center based on the web service (Liu et al.,2006; Fu et al.,2005).

\subsection{System Development Tools}

System development carrys out on the operation systerm of the Windows 2003 enterprise, the used device is ZX-6000 PDA, main tool softwares include Microsoft Visual Studio .NET, Pocket PC SDK, Microsoft ActiveSync 4.5, Microsoft SQL Server 2005 and so on.

\section{RESULT AND ANALYSIS}

\subsection{Setting and Invalidation of Tracing Ear Tags}

According to the Administrative Rules, pigs must be given the unique ID which is weared on one of ears in the first disease immunity. As Fig 3 show, firstly identify ear tags through ear tag reader devices (most continuous ear tags is 15), then enter the information of pig owners and pen No (or group 
No).In the system, the default type of livestock is pig, the reaing purpose is fattening and the default address is the township related to the devices. In case of death or slaughter, the relative ear tag must be Invalidated disposal in order to avoid repeated use (as shown in Fig 3).

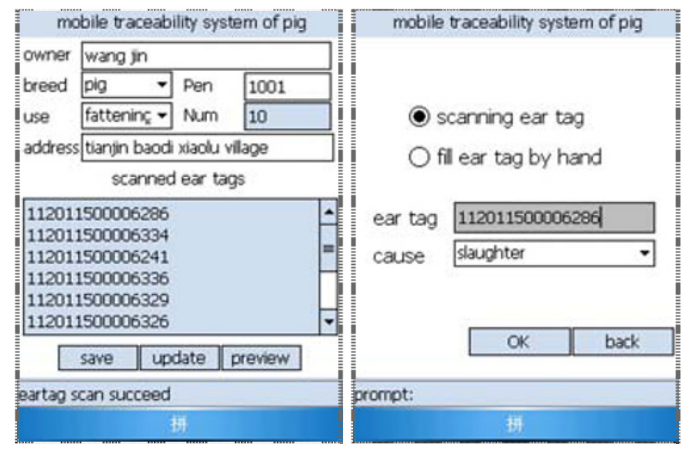

Fig.3 Setting and Invalidation of Pig Ear tags

\subsection{Information Collection of Traceability Inputs}

Information collection of inputs is to collect use informations of vaccines, feeds and veterinary drugs. In general, registration of the epidemic prevention event for vaccines needs to identify one ear tag or more every pen or group of pigs, then the system will create the same event information for all other pigs in the same pen or group. The field of Note is not required to fill out but the others required. When the data uploads, the system automatically loads information about operating deviec ID and operating date (Fig 4).

After registration of the epidemic prevention event, to register for use inforamtions of feeds and veterinary drugs. Registration of the feed event mainly register name,source and use date of pig feeds. Though the Note is
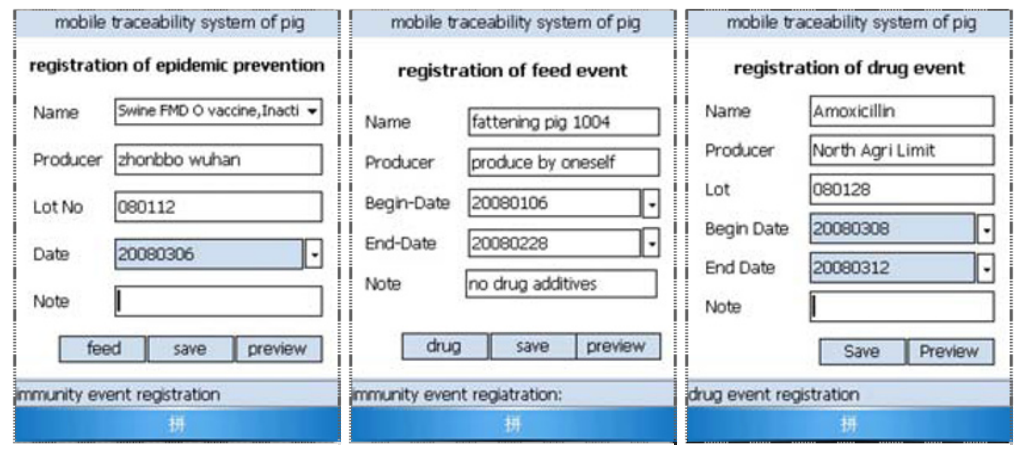

Fig4. Information collection of Input Events 
not required to fill out, but reuqired when the pig feeds have added drug addtives requiring the withdrawal time to achieve the level of unidentified drug residues before sold for slaughter.

Whereas registration of the veterinary drug event mainly record use information of drugs treated for diseases, in general official veterinarians examine the drug event information before sold for slaughter or quarantine in producing area in order to ensure no drug residues in pigs. Note is not required to fill out any information.

When traceability data center receives uploading informations from the client PDA, the system automatically conducts integration process of event inforamtions respectively including epidemic prevention event, veterinary drug event and feed event.

\subsection{Information Collection of Supervision}

The system provides the collection solution for informations produced in the testing process by official veterinarians (Fig 5).In order to simplify operations and avoid operation errors, every event information is divided into detail selection items to fill out. The system automatically updates device ID. Traceability data center will timely receive the data and integrate them into the supervision event.

When a official veterinarian is in the process of monitoring, he can query traceability inforamtion for sampling pigs by his PDA device.

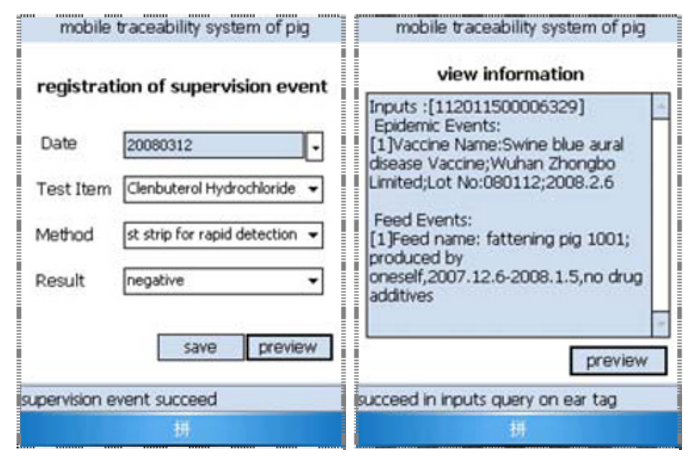

Fig5. Information collection and Query of Superveision Event

\section{DISCUSSION}

After many years' construction of agricultural information collection system, a number of relatively stable channels of information collection have put in practice in areas of agriculture and animal husbandry, but the major information belongs to statistical information after the event. Majority of 
systems are vertically deployed with multi-level, high maintenance costs and slow updating rate. Because rural infrastructure is still developing, the national agricultural information collection system can only be deployed to county levels and above nowadays and is very difficult to every town and village in rural areas. Farmers are lack of abilities and means of access information. The delivery methods of agricultural information is not in good harmony with current widespread used information tools and information use abilities of farmers(Wang,2007). Because this study adopts 2D ear tags and PDA readers for pigs appointed by the Ministry of Agriculture, produced in appointed enterprises, managed and used in scopes of country, the information collection solution will is feasible in extension and application.

This study conducts and realizes mobile PDA collection, edition and transmission of traceability system for smallhold pig farming by using PDA with embedded system.In comparing with internet traceability information systems, data collecting and editing is simple and concise.Event information for various inputs is reasonablly distributed to information collection forms where operations are simple and data volume is low, which not only reduces the workload and operation cost but also meets with demands of pork quality traceability, is a practical and reliable information collection solution by the mobile communication.

Apart from information infrastructure, one more important task is continually to perfect principles of information collection and transmission, and to confirm responsible persons in charge of collection and upload of key traceability informations including setting and invalidation of ear tags, inputs event (vaccines, feed, veterinary drugs) and supervision event. Because official veterinarians is in charge of immunization, diseases treatments and supervision of establishing rearing files for every pig from farms or households on behalf of government, all mobile PDA in the solution are assigned to official veterinarians.All traceability information must be examined and confirmed by official veterinarians who are responsible for authenticity of the informations before updating to the traceability data center.

\section{ACKNOWLEDGEMENTS}

Appreciation is extended to Ma Xing-jian, Beijing PingZhi Dongfang Sci\&Tech Development Limited, for his practical suggestions and helps in process of this system developement. Funding were provided by the national high technology research and development program of China (863 Program) (2006AA10Z266), Tianjin municipal science \& technology Innovation 
fund(06FZZDNC0100) and the national key technology R\&D program (2006BAK02A16).

\section{REFERENCES}

Ammendrup S, Fussel A-E. Legislative requirements for the identification and traceability of farm animals within the European Union[J].Rev sci tech Off in Epiz,2001,20:437-444.

Caja G, Hernandez M, Conill C et al. Use of ear tags and injectible transponders for the identification and traceability of pigs from birth to the end of the slaughter line[J].J Anim Sci,2005,83:2215-2224.

Chen Hong-hua,Tian Zhi-hong. Comparative study on Farm Products Traceability System at Home and Abroad.Market Modernization[J],2007,(21):5-6.

Decree No 67,the Ministraty of Agrilculture of the People's Republic of China. the Administrative Rules on identification and rearing files for animal and poultry. http://www.agri.gov.cn/blgg/t20060628_638621.htm,June 28,2006.

Fu xi,Qi ning,Xu Jun. Mobile Telephone Application and Development of Windows Mobile $[\mathrm{M}]$. Beijing:posts \& telecom press, 2005.

Jill E. Hobbs,Deevon bailey,et al..Traceability in the Canadian red meta sector: do consumers care?.Canadian Journal of Agrcultural Economics , 2005,53: 47-65.

Liu Yan-bo,Hu Yan,Ma qi. Application and Developement of Windows Mobile Platform [M]. Beijing:posts \& telecom press, 2006.

$\mathrm{N}$ schembti, Ja toribio.Review of identification and traceability legislation for pigs in Australia[J].Australian Veterinary Journal , 2007,85(7):255-260.

Wang Xin. Mobile communications technology's application in the agriculture instant information collection and service [J].Agriculture Network Information.2007,(12):14-17.

Xiong ben hai et al. A practical web-based tracking and traceability information system for the pork products supply chain[J]. New Zealand Journal of Agricultural Research ,2007,50:725 733.

Zhang Guoqing. The Framework of Forest Product Traceability System. http://www.scichi.com/new/ duzhe/Article/154.html. 1,16,2008.

Zhang Jing-sheng. Methods of Building Animal Identification Traceability System in the World [J].China Animal Husbandry Bulletin,2006,(7):60-62. 